in der rechten Amygdala korrelierte zudem mit der Abnahme des Alkoholverlangens.

\section{Kommentar}

Diese methodisch sehr hochwertige, doppelblinde, randomisierte Studie zeigt, dass das Cognitive Bias Modification Training die mesolimbische Aktivierung durch Alkoholstimuli bei alkoholabhängigen Patienten reduziert. Damit scheint das Training die erlernte selektive Aufmerksamkeitsfokussierung bei alkoholabhängigen Patienten zu verringern.
Zudem zeigt die Studie, dass die Effekte des Trainings auf die mesolimbische Aktivität mit der Reduktion des Alkoholverlangens korrelieren. Diese Studie deckt damit einen biologischen Mechanismus auf, der die Wirksamkeit von Cognitive Bias Modification Training erklärt.

Die Studie ist mit 32 Teilnehmern aufgrund der Fallzahl limitiert. Dies könnte erklären, wieso die Abnahme der kognitiven Verzerrung in der Interventionsgruppe und die Effekte auf den Nucleus accumbens kein signifikantes Niveau erreicht haben. Besonders hervorzuheben ist, dass in der Studie für die Verhaltens- und Hirnaktivitätsmessungen andere Alkoholstimuli eingesetzt wurden als für das Training, was zeigt, dass das Training auf allgemeine Alkoholreize generalisieren kann.

Zusammengefasst handelt es sich beim Cognitive Bias Modification Training um ein an der Pathologie orientiertes, wirksames und damit klinisch interessantes Verfahren.

PD Dr. med. Bernd Lenz

1. Wiers $C E$ et al. Effects of cognitive bias modification training on neural alcohol cue reactivity in alcohol dependence. Am J Psychiatry $2015 ; 172(4): 335-43$

\title{
Eine internetbasierte Kurzintervention zur Reduktion ungesunden Alkoholkonsums bei jungen Männern
}

Im randomisiert kontrollierten Vergleich reduziert eine internetbasierte Intervention die Anzahl von konsumierten Standardgetränken/Woche und den AUDIT-Score bei jungen Männern mit ungesundem Alkoholkonsumverhalten.

Ü bermäßiger Alkoholkonsum ist bei jungen Männern ein wesentlicher Risikofaktor für Unfälle, Gewalt und spätere Alkoholabhängigkeit. Es gibt Belege dafür, dass Screeningverfahren und Kurzinterventionen die Alkoholkonsummuster deutlich verbessern können. Internetbasierte Angebote sind günstig, rund um die Uhr sowie von zuhause aus verfügbar und ihre Anwendung ist aufgrund der Anonymität nicht stigmatisierend. Einige Studien deuten an, dass internetbasierte Screening- und Kurzinterventionsverfahren übermäßigen Alkoholkonsum reduzieren können.

In der randomisierten kontrollierten Studie [1] wurde der Effekt einer internetbasierten Intervention zur Reduktion ungesunden Alkoholkonsums bei jungen Männern untersucht. Dieser war definiert als $>14$ Standardgetränke/Woche oder $\geq$ eine Binge drinking-Episode/Monat in den vorherigen zwölf Monaten oder ein AUDIT-Score $\geq 8$. Potenzielle Interessenten der schweizerischen „Cohort study on Sub-stance Use Risk Factors" (C-SURF, www.c-surf.ch) wurden per SMS und E-Mail zur Teilnahme eingeladen. Die Anzahl der alkoholischen Standardgetränke pro Woche und Binge drinking-Verhalten wurden vor Studien- beginn, nach einem und nach sechs Monaten erfasst, der AUDIT-Score sowie die Anzahl alkoholbedingter Folgen vor Studienbeginn und nach sechs Monaten. Die Intervention orientierte sich an Inhalten von www.alcooquizz.ch mit allgemeinen Informationen und Empfehlungen zu Alkoholkonsum und Gesundheit, mit Rückmeldung zum individuellen Alkoholkonsum, zu möglichen Folgen übermäßigen Alkoholkonsums, zur Kalorienmenge und zur geschätzten Blutalkoholkonzentration. Die Kontrollgruppe erhielt keine Intervention.

737 junge Männer (im Mittel 20,75 Jahre alt) gaben ungesunden Alkoholkonsum an (im Mittel 9,8 Standardgetränke/Woche, 10,6 Punkte im AUDIT, $84,9 \%$ monatliche Binge drinking-Episoden) und wurden entweder der Intervention ( $\mathrm{n}=367)$ oder der Kontrollbedingung $(n=370)$ zugeteilt. Im SechsMonats-Beobachtungszeitraum kam es in der Interventionsgruppe im Vergleich zur Kontrollgruppe zu einer signifikant stärkeren Reduktion der Anzahl von Standardgetränken/Woche (mittlere Differenz, $-1,68$ vs. $-0,39$; IRR 0,90, $95 \%$-KI: $0,81-0,99)$ und des AUDITScores (mittlere Differenz, $-1,71$ vs. $-0,93$; IRR 0,93, 95\%-KI: 0,88-0,98).
Die Interventionsgruppe zeigte im Vergleich zur Kontrollgruppe keine signifikanten Effekte auf Binge drinking-Episoden oder Anzahl von Alkoholfolgen.

\section{Kommentar}

Das in dieser kontrollierten randomisierten Studie angewandte internetbasierte Interventionsprogramm reduzierte im SechsMonats-Beobachtungszeitraum erfolgreich die Anzahl von konsumierten Standardgetränken pro Woche und den AUDIT-Score bei jungen Männern mit ungesundem Alkoholkonsum. Eine besondere Stärke dieser Studie ist der Zugang über eine populationsbasierte Kohorte und der Einbezug von deutsch- und französischsprachigen Teilnehmern. Eine Limitation ist die digitale Selbsterfassung der Endpunkte, die zum Beispiel im Rahmen sozialer Erwünschtheit zu einer Untertreibung des Konsumausmaßes geführt haben könnte. Da hier nur Männer untersucht wurden, lässt sich nicht abschätzen, ob die Intervention ähnliche Erfolge bei Frauen zeigen würde. Internetbasierte Programme bieten interessante Ansätze in der Therapie ungesunden Alkoholkonsums. Weitere Studien sind notwendig, um die Wirksamkeit unterschiedlicher Inhalte gegeneinander zu validieren.

PD Dr. med. Bernd Lenz

1. Bertholet $\mathrm{N}$ et al. Internet-based brief intervention for young men with unhealthy alcohol use: a randomized controlled trial in a general population sample. Addiction 2015; 10: $1735-43$ 